\title{
INFLUENCE OF THE CALCULATED LENGTH OF ELEMENTS ON THE CRITICAL PARAMETERS OF STABILITY OF FRAME-BAR STRUCTURAL SYSTEMS
}

\section{Emelyanov Sergey} Gennadievich

Southwest State University, Kursk, Russian Federation

\author{
Dubrakov Sergey \\ Valerievich \\ Southwest State University, \\ Faculty of Civil Engineering and \\ Architecture, Department of \\ Industrial and Civil Engineering, \\ Kursk, Russian Federation
}

\author{
Dubrakova Ksenia Olegovna \\ Southwest State University, \\ Faculty of Civil Engineering \\ and Architecture, Department \\ of Industrial and Civil Con- \\ struction, \\ Kursk, Russian Federation
}

Key words: stability, frame-rod system, active bifurcation, passive bifurcation, calculated length doi:10.5937/jaes18-27801

\section{Cite article:}

Gennadievich, S. E., Valerievich, S. D., \& Olegovna K. D. [2020]. Influence of the calculated length of elements on the critical parameters of stability of frame-bar structural systems. Journal of Applied Engineering Science, 18(3) 422 - 426. 


\title{
INFLUENCE OF THE CALCULATED LENGTH OF ELEMENTS ON THE CRITICAL PARAMETERS OF STABILITY OF FRAME-BAR STRUCTURAL SYSTEMS
}

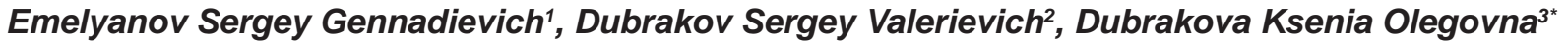 \\ ${ }^{1}$ Southwest State University, Kursk, Russian Federation \\ ${ }^{2}$ Southwest State University, Faculty of Civil Engineering and Architecture, Department of Industrial and \\ Civil Engineering, Kursk, Russian Federation \\ ${ }^{3}$ Southwest State University, Faculty of Civil Engineering and Architecture, Department of Industrial and \\ Civil Construction, Kursk, Russian Federation
}

This article examines the effect of changing the design length of the elements of frame-bar structural systems operating in a constrained bifurcation.

For the criterion of the form of buckling of an element of a frame-bar structural system, the sign of the work of end moments and shear forces is taken. Using this criterion, an expression was obtained to assess the effect of changing the calculated length of the frame struts on the critical parameters of the entire system, which allows varying the geometry of the structure to influence its performance.

A two-span frame is considered, in which the central pillar is loaded with a concentrated force Pcr, and the outer pillars are loaded with forces $\alpha$ Rcr. The type of bifurcation of the rods (constrained or forced) and the critical parameters of the stability of the system before and after changing the calculated length of its elements are determined.

Changes in the design length of elements that passively lose stability do not have a significant effect on the critical stability parameters of the frame-bar structural system. At the same time, a 30\% decrease in the calculated length of the struts in an active bifurcation leads to a decrease in the critical force by $50 \%$.

The presented rather simple algebraic dependencies allow obtaining a qualitative and quantitative assessment of the effect of the calculated length coefficient on the stability of frame-rod structural systems made of wood. It has been established that the conditions for securing elements that passively lose stability do not have a significant effect on the critical parameters of the system.

Key words: stability, frame-rod system, active bifurcation, passive bifurcation, calculated length

\section{INTRODUCTION}

It is known that the reason for the loss of stability of an entire structural system such as a truss, frame, structure is often one element or a small group of them. In this regard, an important issue in solving problems of stability of structural systems is the identification of the most dangerous elements or parts of a structure with low resistance to buckling [1-4, 9-13, 17-19]. Therefore, it is necessary to investigate the deformation of the rods under constrained bifurcation conditions and establish the criteria for the type of rod bifurcation (constrained or forced).

\section{MATERIALS AND METHODS}

Let the bar separated from the system (Fig. 1) at some critical value of the load $P_{c r}(w, t)$ loses stability.

Its deformation energy $U_{i}$ added during bifurcation will be equal to the sum of the work of end forces and moments:

$U_{i}=A_{i}(N)+A_{i}\left(M_{i}, Q_{i}\right)$,

The criterion $A_{i}\left(M_{i}, Q_{i}\right)<0$ ecomes a sign of active buckling of the bar in the structural system, i.e. the work of one longitudinal force $N_{i}$ is sufficient to compensate for the in- crement in the potential energy of deformation of the rod $U_{i}$ and the environment of the rod (in the form of support reactions) to resist its bifurcation.

The criterion $A_{i}\left(M_{i j} Q_{i}\right)>0$ becomes a sign of passive buckling, i.e. surrounding the rod helps its bifurcation.

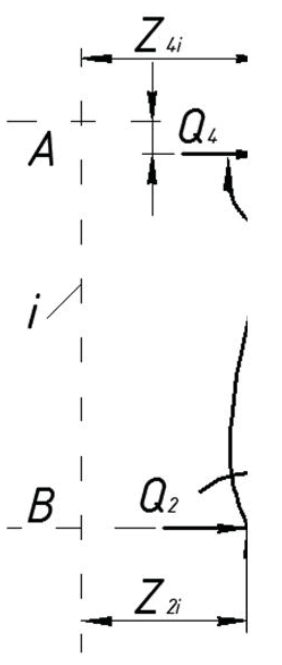

Figure 1: Bar extracted from the system 
The calculation of the work of end moments and shear forces is performed using special functions of the displacement method [5-8, 14-16].

We take for unknown angles of rotation and displacement of nodes $Z_{1}, Z_{2}, Z n$, then the homogeneous system of equations will take the form:

$$
\begin{aligned}
& \begin{aligned}
r_{11} \cdot Z_{1}+ & r_{12} \cdot Z_{1}+\cdots+r_{1 n} \cdot Z_{n} \\
& =0 r_{21} \cdot Z_{1}+r_{22} \cdot Z_{1}+\cdots+r_{2 n} \cdot Z_{n} \\
& \left.=0 \ldots r_{n 1} \cdot Z_{1}+r_{n 2} \cdot Z_{1}+\cdots+r_{n n} \cdot Z_{n}=0\right\}
\end{aligned} \\
& v_{i}=l \cdot \sqrt{\frac{P_{i}}{B_{\text {red }}}},(i=1,2,3 \ldots n) .
\end{aligned}
$$

In formula (3) $B_{\text {red }}$ is the reduced stiffness of the bar section; $v_{i}$ - - parameter of the secular equation.

Determine the determinant of the system (2):

$$
\text { Det }=0
$$

Using the above equations, we find the critical parameters and forms of loss of stability of the considered structural system. Of interest is the assessment of the influence of the loading scheme on the nature of the bifurcation of individual struts.

It is also necessary to analyze the change in the value of the critical force in the event of a change in the calculated length of each of the frame struts. In fact, it can be expected that, under the influence of force factors, a change in the calculated length of the struts, which are losing stability passively and actively, will have a different effect on the overall bifurcation of the system.

To solve this problem, let us determine the parameter of the secular equation $v i_{k p}$, determined after the occurrence of an emergency situation associated with a change in the estimated length of the racks:

$v_{i_{c r}}=l \cdot \sqrt{\frac{P}{B_{\text {red }}}}$

The parameter of the secular equation at a given constant value of the load and a fixed value of wood moisture will be respectively equal to:

$v_{i_{0}}=l \cdot \sqrt{\frac{\mathrm{P}}{B_{0}}}$.

The ratio of the squares of the critical and initial parameters of the secular equation is directly proportional to the ratio of the corresponding critical forces:

$\frac{v_{i_{c r}}{ }^{2}}{v_{i_{0}}{ }^{2}}=\frac{\mathrm{P}_{c r}}{\mathrm{P}_{c r, 0}}$

The parameters $v i_{0}$ and $v i_{c r}$ are determined when calculating the frame for stability according to the well-known rules of structural mechanics before and after changing the calculated length of the racks.

Using relation (7), it is possible to estimate the effect of changing the calculated length of the frame struts on the critical parameters of the entire system, which in

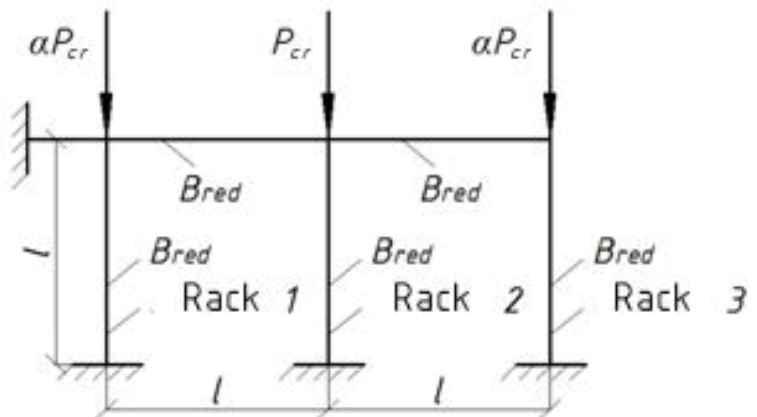

Figure 2: Design scheme of the frame

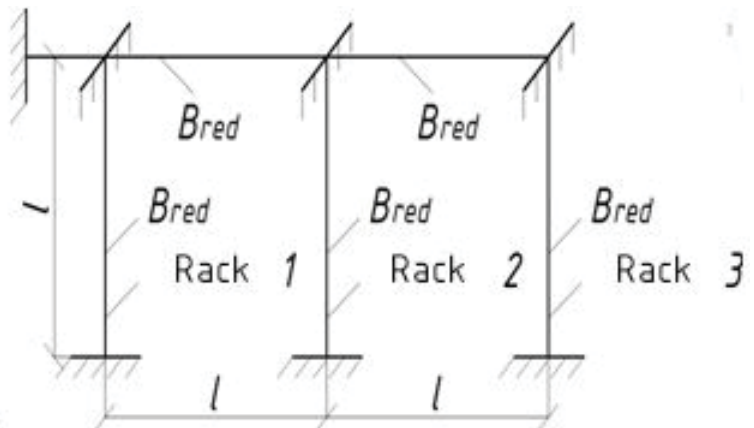

Figure 3: Basic system of the displacement method

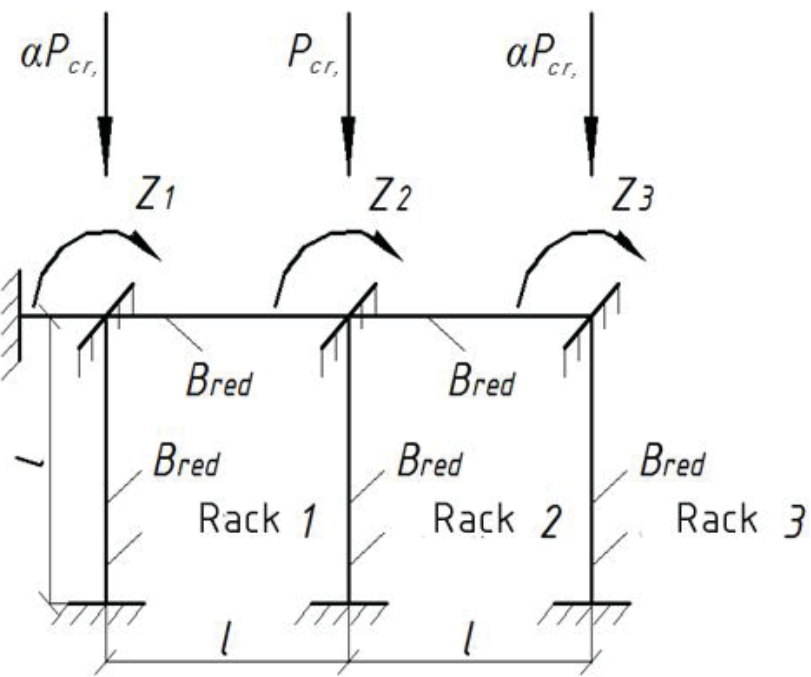

Figure 4: Equivalent displacement method system

turn allows, by varying the geometry of the structure, to influence its performance. The larger the value $v_{i_{c r}}{ }^{2}$, the more likely the frame as a whole will lose stability. $\frac{v_{c r}}{v_{i_{0}}{ }^{2}}$ Results. As an example, consider a wooden two-span frame in which the central pillar is loaded with a concentrated force $P_{c r}$ and the outer pillars are loaded with forces $\alpha P_{c r}$ (Fig. 2).

Let us define the type of the rod bifurcation (constrained or forced). The calculation of the frame is performed by a quasi-static method of displacement using a step-iterative procedure. The calculation of the work of the end moments and shear forces is performed using special functions of the displacement method. If we take as unknown angles of rotation of nodes $Z_{1}, Z_{2}, Z_{3}$ (Fig. 4), the homogeneous system of equations will take the form: 


$$
\begin{gathered}
r_{11} \cdot Z_{1}+r_{12} \cdot Z_{1}+r_{13} \cdot Z_{3}=0 r_{21} \cdot Z_{1}+r_{22} \cdot Z_{1}+r_{23} \cdot Z_{3} \\
\left.=0 r_{31} \cdot Z_{1}+r_{32} \cdot Z_{1}+r_{33} \cdot Z_{3}=0\right\}
\end{gathered}
$$

where

$$
\begin{aligned}
& r_{11}=8 \cdot i+4 \cdot i \cdot \varphi_{2}\left(v_{1}\right) ; \quad r_{22}=8 \cdot i+4 \cdot i \cdot \varphi_{2}\left(v_{2}\right) ; \quad r_{33}=4 \cdot i+4 \cdot i \cdot \varphi_{2}\left(v_{3}\right) ; \\
& r_{12}=r_{23}=2 \cdot i ; \\
& r_{13}=r_{31}=0 ; v_{i}=l \cdot \sqrt{\frac{P_{i}}{B_{\text {red }}(w, t)}}, \quad(i=1,2,3) .
\end{aligned}
$$

where $B_{\text {red }}$ is the reduced stiffness of the bar section; $v_{\mathrm{i}}-$ parameter of the secular equation.

The determinant of system (8) is determined by the following expression:

$$
\begin{gathered}
\text { Det }=\left(8 \cdot i+4 \cdot i \cdot \varphi_{2}\left(v_{1}\right)\right) \cdot\left(8 \cdot i+4 \cdot i \cdot \varphi_{2}\left(v_{2}\right) \cdot\left(4 \cdot i+4 \cdot i \cdot \varphi_{2}\left(v_{3}\right)\right)+16 \cdot i^{3}\right. \\
-\left(8 \cdot i+4 \cdot i \cdot \varphi_{2}\left(v_{1}\right)\right) \cdot 4 \cdot i^{2}-4 \cdot i^{2} \cdot\left(4 \cdot i+4 \cdot i \cdot \varphi_{2}\left(v_{3}\right)\right)
\end{gathered}
$$

Using the above equations, we find the critical parameters and forms of loss of stability of the considered structural system.

It is also necessary to analyze the change in the value of the critical force in the event of a change in the calculated length of each of the frame struts. In fact, it can be expected that, under the influence of force factors, a change in the calculated length of the struts, which are losing stability passively and actively, will have a different effect on the overall bifurcation of the system.

To solve this problem, let us determine the parameter of the secular equation $v i_{c r}$, determined after the occurrence of an emergency situation associated with a change in the estimated length of the racks:

$v_{i_{c r}}=l \cdot \sqrt{\frac{P}{B_{\text {red }}}}$

The parameter of the secular equation at a given constant value of the load and a fixed value of wood moisture will be respectively equal to:

$v_{i_{0}}=l \cdot \sqrt{\frac{\mathrm{P}}{B_{0}}}$.

The ratio of the squares of the critical and initial parameters of the secular equation is directly proportional to the ratio of the corresponding critical forces:

$\frac{v_{i_{c r}}^{2}}{v_{i_{0}}{ }^{2}}=\frac{\mathrm{P}_{c r}}{\mathrm{P}_{c r, 0}}$.

The parameters $v i_{0}$ and $v i_{c r}$ are determined when calculating the frame for stability according to the well-known rules of structural mechanics before and after changing the calculated length of the racks.

Using relation (12), it is possible to estimate the effect of changing the calculated length of the frame struts on the critical parameters of the entire system, which in turn allows, by varying the geometry of the structure, to influence its performance. The larger the value $\frac{v_{i_{c r}}{ }^{2}}{v_{i_{0}}{ }^{2}}$ the more likely the frame as a whole will lose stability.

Consider the change in the calculated lengths of each of the frame struts, replacing the rigid support with a hinged-fixed one. The design diagram of the frame for this case is shown in Figure 5.

The based and equivalent systems of the displacement method are developed similarly to those presented above (see Fig. 3, 4). The homogeneous system of equations will take the form:

$$
\begin{gathered}
\begin{aligned}
r_{11} \cdot Z_{1}+r_{12} \cdot & Z_{1}+r_{13} \cdot Z_{3} \\
= & 0 r_{21} \cdot Z_{1}+r_{22} \cdot Z_{1}+r_{23} \cdot Z_{3} \\
& \left.=0 r_{31} \cdot Z_{1}+r_{32} \cdot Z_{1}+r_{33} \cdot Z_{3}=0\right\}
\end{aligned} \\
r_{11}=8 \cdot i+4 \cdot i \cdot \varphi_{1}\left(v_{1}\right) ; r_{22}=8 \cdot i+4 \cdot i \cdot \varphi_{2}\left(v_{2}\right) ; \\
r_{33}=4 \cdot i+4 \cdot i \cdot \varphi_{2}\left(v_{3}\right) ; r_{12}=r_{23}=2 \cdot i ;
\end{gathered}
$$

where

$$
\begin{aligned}
& r_{13}=r_{31}=0 ; v_{i}=l \cdot \sqrt{\frac{P_{i}}{B_{\text {red }}(w, t)}}, \\
& (i=1,2,3) .
\end{aligned}
$$

The determinant of system (13) is determined by the expression:

$$
\begin{aligned}
\operatorname{Det}=(8 & \left.\cdot i+4 \cdot i \cdot \varphi_{1}\left(v_{1}\right)\right) \cdot\left(8 \cdot i+4 \cdot i \cdot \varphi_{2}\left(v_{2}\right)\right. \\
& \cdot\left(4 \cdot i+4 \cdot i \cdot \varphi_{2}\left(v_{3}\right)\right)+16 \cdot i^{3}- \\
& \left(8 \cdot i+4 \cdot i \cdot \varphi_{1}\left(v_{1}\right)\right) \cdot 4 \cdot i^{2}- \\
& 4 \cdot i^{2} \cdot\left(4 \cdot i+4 \cdot i \cdot \varphi_{2}\left(v_{3}\right)\right) .
\end{aligned}
$$

Similarly, the calculation of the change in the critical stability parameters of the considered frame-bar structural system (see Fig. 2) was performed when the calculated lengths of the racks 2 and 3 were changed.

The results of this calculation are shownin Figure 6 . The vertical lines limit the zones of active and passive bifurcation of racks 1 and 3 , rack 2 actively loses its stability, regardless of the value of the load application parameter $\alpha$.

From the analysis of Figure 6 , it can be concluded that with a value of $\alpha \leq 0.756$, the left and right struts of the structural system passively lose their stability. In the case when the 


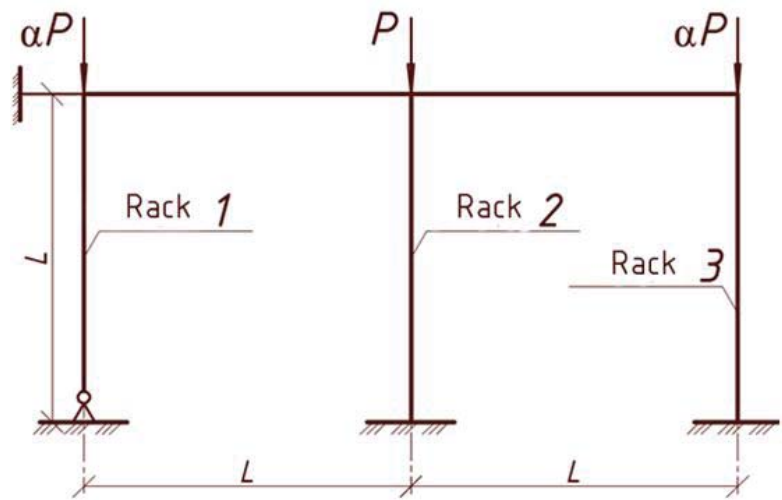

Figure 5: Design scheme of the frame when changing the design length of the rack 1

load application parameter a exceeds the values of 0.756 , the post 3 of the frame under consideration goes over to active bifurcation, with $\alpha>0.9$, all the posts lose their stability actively. At the same time, with a value of $a \leq 0.9$, when the post 1 passively loses its stability, a change in its calculated length leads to a decrease in the critical force by an average of $15 \%$. In the case when the load application parameter $\alpha$ exceeds the value of 0.9 , a change in the calculated length of element 1 leads to an increase in the maximum value of the compressive force by $5 \%$.

At the same time, at a value of $a \leq 0.76$, when the post 3 loses its stability passively, a change in its calculated length leads to a decrease in the critical force by an average of $6 \%$. In the case when the load application parameter a exceeds the value of 0.76 , a change in the calculated length of element 3 leads to an increase in the maximum value of the compressive force by $2 \%$.

In this case, a change in the calculated length of the second leg leads to a decrease in the critical force by an average of $50 \%$

\section{EXPERIMENTAL RESEARCH}

In order to test the developed methodology on the basis

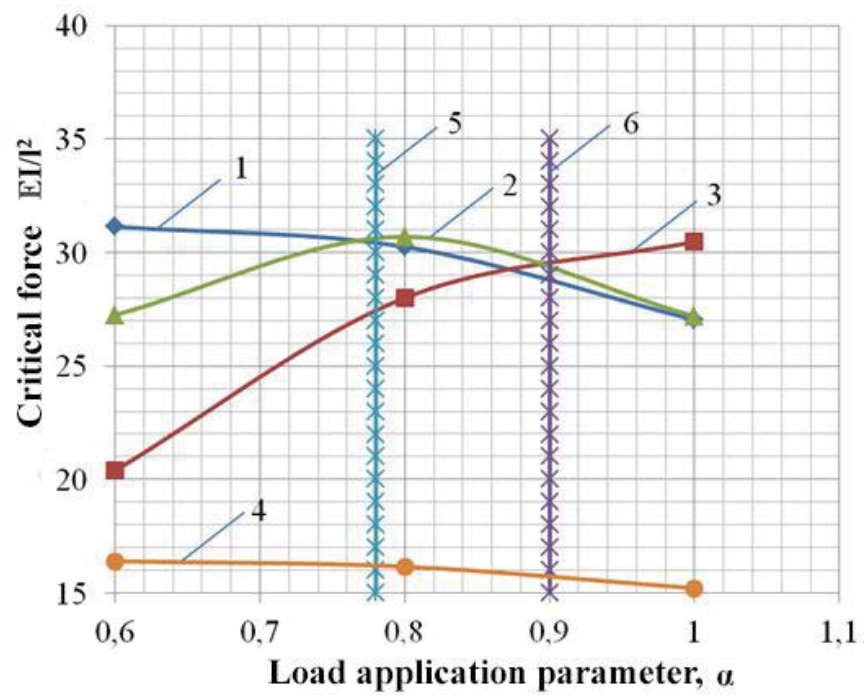

Figure 6: Graph of the dependence of the value of the critical force $P_{\text {cr.o }}$ on the load application parameter $\alpha$ when changing the calculated length of the struts of the frame-rod system, indicating the nature of the bifurcation:

1 - the calculated length of all frame elements is the same, 2 - a decrease in the calculated length of the rack 3 by 30\%; 3 - reduction of the estimated length of the rack 1 by 30\%; 4 - reduction of the estimated length of the rack 2 by $30 \%$; 5 - border of transition of rack 3 from passive loss of stability to active; 6 - border of the transition of rack 1 from passive to active buckling

of the Southwestern State University, experimental studies of the influence of the calculated length on the stability of frame-rod structural systems made of wood were carried out. To achieve this goal, three series of three frames in each were designed with the following parameters: material - spruce wood, grade I, rack height - $1 \mathrm{~m}$; spans - $0.67 \mathrm{~m}$ (Fig. 7). The calculated length was varied due to the rigidity of the support nodes. In the first series, all frame units are

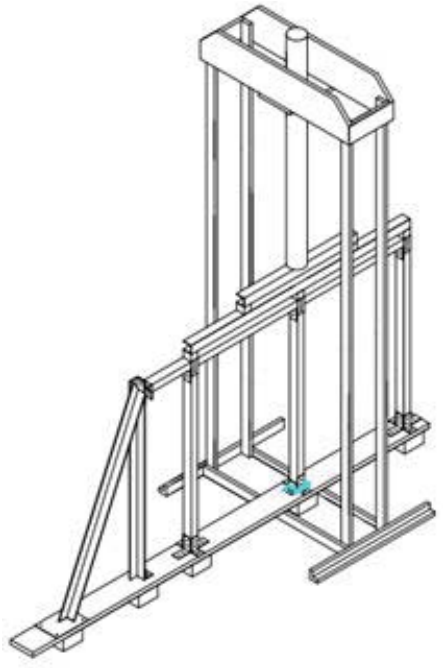

a)

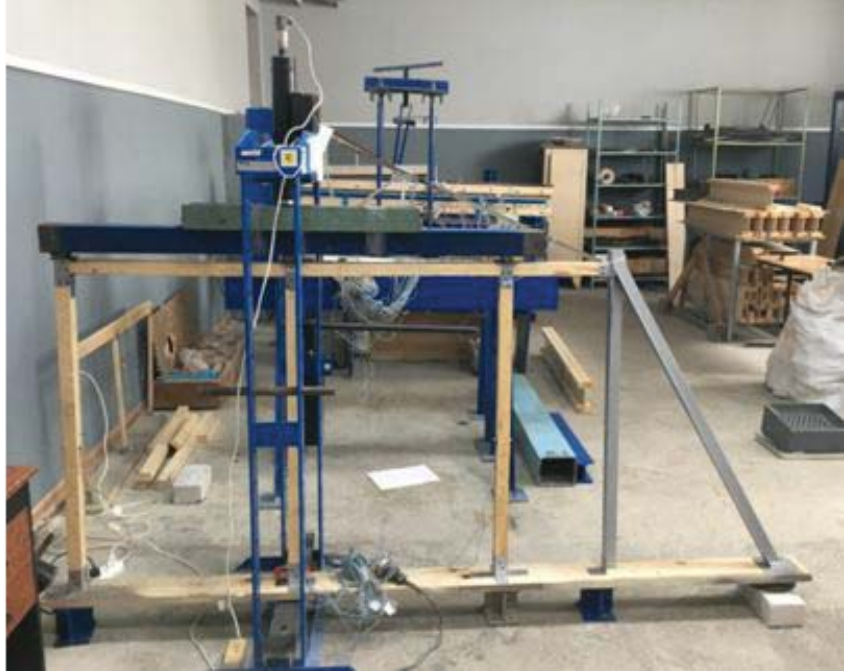

b)

Figure 7: Scheme (a) and general view (b) of the test setup 
rigid; in the second series of hinged-fixed, the support of the left pillar was adopted, in the third - the right one. The tests were carried out with a loading coefficient $\alpha=0.85$, therefore, in the second group of structures, the calculated length of the rack, which loses its stability actively, changes, in the third - passively.

As a result of the experiment, after statistical data processing, it was found that the critical force Pcr. for frames of the first series it is $4.23 \mathrm{kN}$, for the second $-3.09 \mathrm{kN}$, for the third $-4.19 \mathrm{kN}$.

\section{CONCLUSION}

The presented rather simple algebraic dependences make it possible to obtain a qualitative and quantitative assessment of the influence of the calculated length coefficient on the stability of frame-rod structural systems made of wood. At the same time, it was found that the conditions for fixing elements experiencing passive bifurcation do not significantly affect the overall stability of the system.

1. The Department of Construction Sciences RAASN. 2004. - Issue. 8. - S. 14-21.

2. Aleksandrov, AV Criteria for identifying the most dangerous elements and their use in problems of structural stability / AV Aleksandrov, AV Matveev // Safety of train traffic: tr. 4th scientific-practical. conf. - M .: MIIT, 2003. - S. III-1 - III-2.

3. Aleksandrov, A. V. About the calculation of rod structures for stability / A. V. Aleksandrov, V. I. Travush, A. V. Matveev // Promyshlennoe i grazhdanskoe stroitel'stvo. - 2002. - No. 3. - P. 16-20.

4. Aleksandrov, A. V. The role of individual elements of the rod system at loss of stability / A. V. Aleksandrov // Bulletin of MIIT. - 2001. - Issue. 5. - P. 46.

5. Dmitrieva, K.O. Issues of stability of rod elements of structural systems made of wood under power and environmental loading / N.V. Klyuev, K.O. Dmitrieva // Construction and reconstruction. - 2016. - No. 4. S. 13-18.

6. Dubrakova, K.O. Issues of stability of statically indeterminate wood systems / K.O. Dubrakova // BST: Bulletin of construction equipment. - 2018. - No. 11. S. 54-55.

7. Klyueva, N.V. Analysis of the stability of timber structures under power loading and variable humidity / N.V. Klyuev, K.O. Dmitrieva // Scientific Bulletin of the Voronezh State University of Architecture and Civil Engineering. Construction and architecture. - 2016. - No. 3. S. 17-24.

8. Klyueva, N.V. Issues of stability of rod elements of structural systems made of wood of various species under power and environmental loading in conditions of high humidity / N.V. Klyuev, K.O. Dmitrieva // Construction and reconstruction. - 2016. - No. 5. S. 60-68.

9. Matveev, A. V. Some issues of creating a specialized software complex for the analysis of bridge structures / A. V. Matveev // MIIT Bulletin. - 2002. - Issue. 7. - P. 76-83
10. Pyatikrestovsky, KP Nonlinear deformations of statically indeterminate wooden structures. Pyatikrestovsky, Kh.S. Khuganov // News of higher educational institutions. Building. - 2013. - No. 11-12. - S. 21-30.

11. Pyatikrestovsky, K. P. Nonlinear methods of mechanics in the design of modern wooden structures / K. P. Pyatikrestovsky. - M .: MGSU, 2014 .-- 320 p.

12. Pyatikrestovsky, K. P. On programming a nonlinear method for calculating wooden structures / K. P. Pyatikrestovsky, V. I. Travush // Academia. Architecture and construction. - 2015. - No. 2. - P. 115-119.

13. Smorchkov, A.A. Safety of structures made of glued wood at the manufacturing stage / A.A. Smorchkov, D.A. Orlov, S.A. Kereb, K.O. Baranovskaya // Industrial and civil construction. - 2013. - No. 12. S. 74-75.

14. Travush, V.I. Long-term strength and stability of compressed wood rods / V.I. Travush, V.I. Kolchunov, K.O. Dmitrieva // Construction and reconstruction. - 2015. No. 5. S. 40-46.

15. Travush, V.I. Stability of compressed wood rods with simultaneous manifestation of power and environmental impact / V.I. Travush, V.I. Kolchunov, K.O. Dmitrieva // Building mechanics and calculation of structures. - 2016. - No. 2. S. 50-53.

16. Travush, V.I. Experimental and theoretical study of the strength and stability of compressed wood rods under power and environmental impact / V.I. Travush, V.I. Kolchunov, K.O. Dmitrieva // News of higher educational institutions. textile industry technology. - 2016. - No. 3. S. 280-285.Dubrakova K. O. The buckling of the physically nonlinear frame-rod structural systems/Dubrakova, K.O., Dubrakov, S.V., Altuhov, F.V., Galaeva, D.H.// 2019 IOP Conf.: Mater. Sci. Eng. 698022007.

17. Yemelyanov-Gennadyevich, S., Gennadievna-Pakhomova, E., Olegovna-Dubrakova, K., \& Valerievich-Dubrakov, S. [2019]. Stability of statically indefinite physically nonlinear timber structural systems. Journal of Applied Engineering Science, 17(3), 404-407.

18. Gennadyevich, Y. S., Pakhomova, E. G., \& Olegovna, D. K. [2019]. Reliability of RC frame-braced systems in dangerous geological conditions. Journal of Applied Engineering Science, 17(2), 245-250.

19. Travush, V.I. Experimental and Theoretical Research of Durabilities and Stability the Squeezed Cores from Wood at Power and Environmental Influence/ Travush, V.I., Kolchunov, V.I., Dmitrieva, K.O.// Proceedings of higher educational institutions. Textile industry technology. 2016 (3): 280-285.

Paper submitted: 04.08.2020.

Paper accepted: 07.09.2020.

This is an open access article distributed under the CC BY 4.0 terms and conditions. 